\title{
Effect of all-trans-retinoic acid on enterovirus 71 infection in vitro
}

\author{
Siyuan Chent, Yi Yangt, Jin Xu, Liyun Su and Weiping Wang* \\ Department of Pediatrics Institute, Children's Hospital of Fudan University, 399 Wanyuan Road, Shanghai 201102, \\ People's Republic of China
}

(Submitted 11 July 2013 - Final revision received 14 November 2013 - Accepted 25 November 2013 - First published online 4 February 2014)

\begin{abstract}
Our previous studies have shown that vitamin A (VA) status is associated with antiviral immunity and pathogenic conditions in enterovirus 71 (EV71)-infected children. In the present study, we established an in vitro model to investigate the effects and potential mechanism of the antiviral activity of VA. Human monocytic U937 cells were cultured in vitro and infected with EV71. All-trans-retinoic acid (ATRA), the active metabolite of VA, and Ro 41-5253, a retinoic acid receptor- $\alpha$ (RAR- $\alpha$ ) antagonist, were used as the experimental treatment agents. The percentage of EV71-infected cells and apoptosis induced by EV71 were determined using flow cytometry. The level of interferon- $\alpha$ (IFN- $\alpha)$ in the supernatants of the cultures was detected using ELISA. The expression of retinoid-induced gene I (RIG-I) and its downstream genes was examined with real-time quantitative PCR. The results indicated that ATRA reduced the percentage of EV71-infected cells and protected cells against EV71-induced apoptosis. Correspondingly, ATRA increased the production of IFN- $\alpha$ one of the most important antiviral cytokines, at both mRNA and protein levels in EV71-infected cells. In addition, the expression of RIG-I mRNA and its downstream genes was up-regulated by ATRA in EV71-infected cells. Ro 41-5253 abrogated the inhibitory effects of ATRA on EV71. The present findings suggest that ATRA is an interferon-inducing agent with antiviral activity against EV71 in vitro and that its actions are mediated at least in part by RAR- $\alpha$ activity and the RIG-I signalling pathway.
\end{abstract}

Key words: All-trans-retinoic acid: Interferons: Retinoic acid receptors: Retinoid-induced gene I

Hand, foot and mouth disease remains a major cause of morbidity and mortality in children in developing countries and the main causative pathogen is enterovirus 71 (EV71). In mainland China, large outbreaks of EV71-caused hand, foot and mouth disease, resulting in millions of cases and thousands of deaths in children, have been reported since $2008^{(1)}$. Furthermore, the cumulative data indicate an increased incidence of hand, foot and mouth disease during 2009 and 2011; at that time, EV71 was still the predominant pathogen $^{(2-4)}$. As yet, there is no effective vaccine to prevent EV71 infection. Without an effective antiviral treatment, patients must rely on their own immune system to overcome the infection.

Vitamin A (VA) is one of the most important nutrients for childhood nutrition. Many epidemiological and clinical investigations have confirmed that VA can boost immunity against infectious diseases in children ${ }^{(5)}$. Previous studies carried out by our group have reported that most of the EV71-infected children present with VA insufficiency, which is associated with decreased immunity and more severe pathogenic conditions $^{(6)}$. Specifically, we found serum interferon- $\alpha$ (IFN- $\alpha$ ) levels to be markedly reduced and positively related to the lack of VA in EV71-infected children ${ }^{(6)}$. These clinical findings stimulated us to establish an in vitro model to investigate the potential mechanism for the effect of VA on antiviral immunity to EV71 infection. Retinoid has been reported to inhibit the replication of various viruses, including the measles virus, the herpes virus, the immunodeficiency virus type 1 and the Epstein-Barr virus, in vitro ${ }^{(7-10)}$. However, there is no report regarding the antiviral effect of retinoid on EV71 in vitro.

The active VA metabolite all-trans-retinoic acid (ATRA) is the natural ligand for the retinoic acid receptors (RAR). There is a rapidly expanding body of literature that supports a role for ATRA in the IFN signalling pathway. In various in vitro systems, ATRA has been shown to regulate the expression of a number of IFN-stimulated genes, including retinoid-induced gene I $(R I G-I)^{(11-13)}$. RIG-I is a patternrecognition receptor involved in the innate immune response of the host. The inhibition of RIG-I-mediated type I IFN

Abbreviations: ATRA, all-trans-retinoic acid; EV71, enterovirus 71; FBS, fetal bovine serum; IFN- $\alpha$, interferon- $\alpha$; RAR- $\alpha$, retinoic acid receptor- $\alpha$; $R I G-I$, retinoid-induced gene I; Ro 41-5253, a retinoic acid receptor- $\alpha$ antagonist; VA, vitamin A.

* Corresponding author: Professor W. Wang, fax +86 21 64931883, email wpwang@fudan.edu.cn

†These two authors contributed equally to the present study. 
responses may contribute to the pathogenesis of EV71 infection $^{(14)}$.

In the present study, we established an in vitro model to test our hypothesis that ATRA can act against EV71 through an IFN-inducing effect, specifically by regulating $R I G-I$ mRNA expression. We used U937 cells, a monocytic cell line, as our model. U937 cells were chosen because previous studies have demonstrated that EV71 can infect human monocytes ${ }^{(15)}$. In addition, U937 cells have also been used to demonstrate that ATRA can influence elements of the type I IFN response ${ }^{(16)}$.

\section{Experimental methods \\ Cell culture and reagents}

The human monocytic cell line U937 (Chinese Academy of Sciences) was maintained in Roswell Park Memorial Institute (RPMI) 1640 (GIBCO/BRL Life Technologies) containing $2 \mathrm{~mm}$-L-glutamine and $10 \%$ fetal bovine serum (FBS) (GIBCO/BRL Life Technologies). African green monkey kidney (Vero) cells (Chinese Academy of Sciences) were maintained in modified Eagle's medium (GIBCO/BRL Life Technologies) with $10 \% \mathrm{FBS}$. All cultures were maintained at $37^{\circ} \mathrm{C}$ in a $\begin{array}{llll}5 \% & \mathrm{CO}_{2} & \text { humidified incubator (Thermo Scientific). ATRA }\end{array}$ (Sigma-Aldrich) and RAR- $\alpha$ antagonist (Ro 41-5253) (Enzo Life Sciences) were kept as stock solutions at $10^{-2} \mathrm{M}$ in $100 \%$ dimethyl sulphoxide (Invitrogen), and further dilutions were made using RPMI 1640.

\section{Virus preparation and titration}

EV71 isolated from a patient's throat swab was provided by the virology laboratory of Children's Hospital of Fudan University. The virus was identified by reverse-transcription PCR and sequencing ${ }^{(17)}$. The virus was propagated in Vero cells in modified Eagle's medium containing 2\% FBS. Briefly, monolayers of Vero cells were inoculated with the virus at a multiplicity of infection of 1 , and the virus culture medium was harvested after incubation for $24 \mathrm{~h}$. The virus titre was determined by measuring the tissue culture infectious dose $\left(\mathrm{TCID}_{50}\right.$ ) values. Serially diluted virus samples (from $10^{-1}$ to $10^{-9}$ ) were added to Vero cells in ninety-six well plates, and four replicate samples were used at each dilution. The ninety-six-well plates were incubated for $7 \mathrm{~d}$ at $37^{\circ} \mathrm{C}$, and the TCID $_{50}$ values were obtained by determining the cytopathic effects of infected Vero cells. A mock control was prepared in the same manner as the EV71 preparation, except that the Vero cells were not infected with EV71.

\section{U937 cell virus infection}

U937 cells were infected with EV71 at a multiplicity of infection of 10 for $2 \mathrm{~h}$ at $37^{\circ} \mathrm{C}$. Following infection, the cells were resuspended at a density of $1 \times 10^{6}$ cells $/ \mathrm{ml}$ in RPMI 1640 containing a reduced concentration of FBS (2\%) in a twenty-four-well plate $(1 \mathrm{ml} /$ well). EV71-infected cells were incubated for $24 \mathrm{~h}$ or $48 \mathrm{~h}$ in the presence of ATRA and/or
Ro 41-5253. U937 cells treated with 0·1\% dimethyl sulphoxide in RPMI with $2 \%$ FBS were used as the mock treatment cells in all the experiments.

\section{Immunofluorescence staining of enterovirus 71-infected cells}

EV71-infected and control cells were placed on glass slides, fixed in $4 \%$ paraformaldehyde for $15 \mathrm{~min}$ and then blocked in $10 \%$ normal goat serum (Vector Laboratories) in PBS (GIBCO/BRL Life Technologies) containing $0 \cdot 1 \%$ Triton X-100 (Sigma-Aldrich) for $25 \mathrm{~min}$ at room temperature. After washing twice with $1 \%$ normal goat serum in PBS, the cells were stained with a 1:500 dilution of a monoclonal antibody against EV71 (Millipore Corporation) overnight at $4^{\circ} \mathrm{C}$. The cells were then stained with a 1:200 dilution of fluorescein isothiocyanate-conjugated secondary antibodies (Santa Cruz Biotechnology) and counterstained with 4,6-diamidino-2-phenylindole dihydrochloride (Vector Laboratories), after which they were observed under a fluorescent microscope (Leica Microsystems).

\section{Enterovirus 71-infected cell count by flow cytometry}

A minimum of $1 \times 10^{6}$ cells were collected by centrifugation at $1200 \mathrm{~g}$ for $5 \mathrm{~min}$ and fixed in $4 \%$ paraformaldehyde (SigmaAldrich) for $15 \mathrm{~min}$. Next, $50 \mu \mathrm{l}$ of permeabilisation medium (Invitrogen) and a 1:200 dilution of a monoclonal antibody against EV71 (Millipore Corporation) were added to each cell pellet, followed by incubation for $1 \mathrm{~h}$ at room temperature. Fluorescein isothiocyanate-conjugated secondary antibodies (Santa Cruz Biotechnology) at a 1:100 dilution were added, followed by incubation for $30 \mathrm{~min}$ in the dark at room temperature. Finally, after three washes with PBS, the cells were resuspended in $0.5 \mathrm{ml}$ of PBS for flow cytometry (BD FACSCalibur; BD Biosciences).

\section{Apoptosis analysis}

The Annexin V-fluorescein isothiocyanate/Propidium Iodide Apoptosis Detection Kit (Merck) was used in accordance with the manufacturer's protocol. After 24 or $48 \mathrm{~h}$ of EV71 and/or ATRA treatment, the cells were harvested and resuspended in binding buffer. Fluorescence signals were detected using a flow cytometer (BD FACSCalibur) within $1 \mathrm{~h}$.

\section{Detection of interferon- $\alpha$ in supernatants}

The level of IFN- $\alpha$ in the supernatants of the cultures was detected after 24 and $48 \mathrm{~h}$ of incubation with each treatment agent, using an ELISA kit (eBioscience). The optical density was detected at a wavelength of $450 \mathrm{~nm}$ on a microplate reader (Wellscan MK3; Labsystems). A standard curve ranging from $7 \cdot 8$ to $500 \mathrm{pg} / \mathrm{ml}$ was constructed using serial dilutions of a human IFN- $\alpha$ standard provided with the kit. Samples with values greater than the negative control but less than $7.8 \mathrm{pg} / \mathrm{ml}$ were assigned values based on the extrapolation of the standard curve. 
Table 1. Primer sequences, annealing temperatures and product sizes in real-time PCR

\begin{tabular}{|c|c|c|c|c|}
\hline \multirow[b]{2}{*}{ Genes } & \multicolumn{4}{|c|}{ Primers $\left(5^{\prime} \rightarrow 3^{\prime}\right)$} \\
\hline & Forward & Reverse & Annealing temperature $\left({ }^{\circ} \mathrm{C}\right)$ & Product (bp) \\
\hline$R A R-\alpha$ & CTGTGAGAAACGACCGAAAC & TTGTCCCAGAGGTCAATGTC & 58 & 211 \\
\hline$I F N-\alpha 1$ & GCCTCGCCCTTTGCTTTACT & CTGTGGGTCTCAGGGAGATCA & 60 & 89 \\
\hline IFNAR1 & AACAGGAGCGATGAGTCTGTC & TGCGAAATGGTGTAAATGAGTCA & 60 & 226 \\
\hline IPS-1 & CAGGCCGAGCCTATCATCTG & GGGCTTTTGAGCTAGTTGGCA & 61 & 168 \\
\hline RIG-I & TGTGCTCCTACAGGTTGTGGA & CACTGGGATCTGATTCGCAAAA & 63 & 120 \\
\hline TRAF3 & GCGTGTCAAGAGAGCATCGTT & GCAGATGTCCCAGCATTAACT & 60 & 133 \\
\hline TBK1 & GGATCACTGCCATTTAGACCC & CAGGCATGTCTCCACTCCAG & 62 & 145 \\
\hline IRF3 & AGAGGCTCGTGATGGTCAAG & AGGTCCACAGTATTCTCCAGG & 61 & 100 \\
\hline$\beta$-Actin & CACCAACTGGGACGACAT & TCTGGGTCATCTTCTCGC & 55 & 138 \\
\hline
\end{tabular}

$R A R-\alpha$, retinoic acid receptor $\alpha$; IFN- $\alpha$, interferon- $\alpha 1$; IFNAR1, type I IFN receptor; IPS-1, IFN promoter-stimulating factor 1 ; RIG-I, retinoid-induced gene I; TRAF3, TNF receptor-associated factor 3; TBK1, TRAF family member-associated NF-кB activator-binding kinase 1; IRF3, interferon regulatory factor 3.

\section{RNA isolation and real-time $P C R$}

The total RNA of the U937 cells was isolated using TRIzol (Invitrogen) according to the manufacturer's instructions. The concentration of RNA was measured by absorbance at $260 \mathrm{~nm}$ in a spectrophotometer (NanoVue; GE Healthcare Bio-Sciences $\mathrm{AB}$ ). Reverse transcriptions were conducted using $1 \mu \mathrm{g}$ of total RNA in a $20 \mu \mathrm{l}$ final volume using the Prime Script ${ }^{\mathrm{TM}}$ RT Reagent Kit (TaKaRa Biotechnology). Realtime PCR was carried out with a Mastercycler realplex 4 PCR system (Eppendorf) using the $\mathrm{RT}^{2}$ SYBR Green qPCR MasterMix (Toyobo) under the following conditions: $95^{\circ} \mathrm{C}$ for $1 \mathrm{~min}$, followed by forty cycles of $95^{\circ} \mathrm{C}$ for $15 \mathrm{~s}, 60^{\circ} \mathrm{C}$ for $15 \mathrm{~s}$ and $72^{\circ} \mathrm{C}$ for $45 \mathrm{~s}$. The relative expression levels of the genes were normalised to those of the housekeeping gene $\beta$-actin using the comparative threshold cycle $\left(C_{t}\right)$ method, and data were analysed using the $\Delta \Delta C_{\mathrm{t}}$ method. Primer sequences, PCR product sizes and annealing temperatures are summarised in Table 1.

\section{Statistical analysis}

The results are presented as means and standard deviations. Statistical operations were conducted with the SPSS Statistical Package, version 13.0. The statistical analysis was conducted using Student's $t$ test for the comparison of means. A probability value $<0.05$ was considered significant.

\section{Results}

\section{All-trans-retinoic acid decreased the percentage of enterovirus 71-infected cells}

The EV71 viral capsid protein $1(\mathrm{VP} 1)^{+}$capsid proteins were detected with an anti-VP1 antibody using immunofluorescence microscopy. $\mathrm{VP}^{+}$proteins were observed in the cytoplasmic region of $\mathrm{U} 937$ cells inoculated with EV71, but not in the mock-infected control cells (Fig. 1). These findings demonstrate that U937 cells can be infected with EV71.

To determine whether ATRA could inhibit EV71 infection in our model, the percentage of infected cells was determined by flow cytometry after staining the cells with an antiEV71 monoclonal antibody. Following $24 \mathrm{~h}$ of incubation, the percentage of EV71-infected cells in the ATRA+EV71 group was lower than that of cells in the EV71 group, but this difference did not reach statistical significance. Importantly, ATRA treatment markedly decreased the percentage of EV71-infected cells $48 \mathrm{~h}$ after infection ( $P<0 \cdot 01$; Fig. 2). Because our previous studies ${ }^{(18-20)}$ indicated that RAR- $\alpha$ is a major mediator of ATRA action, we sought to determine whether the antiviral effect of ATRA requires RAR- $\alpha$ signalling. The addition of Ro 41-5253 to the EV71-infected U937 cell culture significantly reduced EV71 inhibition mediated by ATRA (Fig. 2). These findings provide evidence that ATRA contributes to the defence against EV71 infection in U937 cells and RAR- $\alpha$ is likely to play an important role in this antiviral effect.
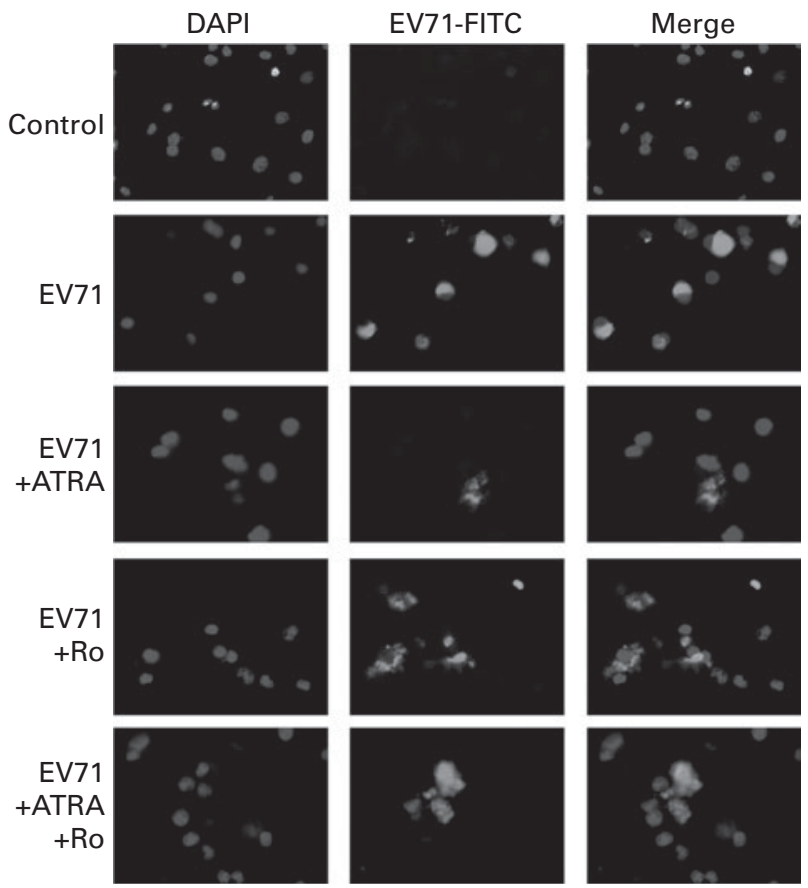

Fig. 1. Immunofluorescent staining of enterovirus 71 (EV71)-infected U937 cells with an anti viral capsid protein 1 (VP1) antibody. U937 $\left(1 \times 10^{6}\right.$ per ml) cells were infected with EV71 at a multiplicity of infection of 10 in the presence of $1 \mu \mathrm{M}$ - all-trans-retinoic acid (ATRA) and/or $10 \mu \mathrm{M}$-Ro 41-5253 (retinoic acid receptor- $\alpha$ antagonist) for $24 \mathrm{~h}$. EV71 antigen (green fluorescence) was observed in the cytoplasmic region of the EV71-infected cells. DAPI, 4,6-diamidino2-phenylindole dihydrochloride; FITC, fluorescein isothiocyanate. 
(a)
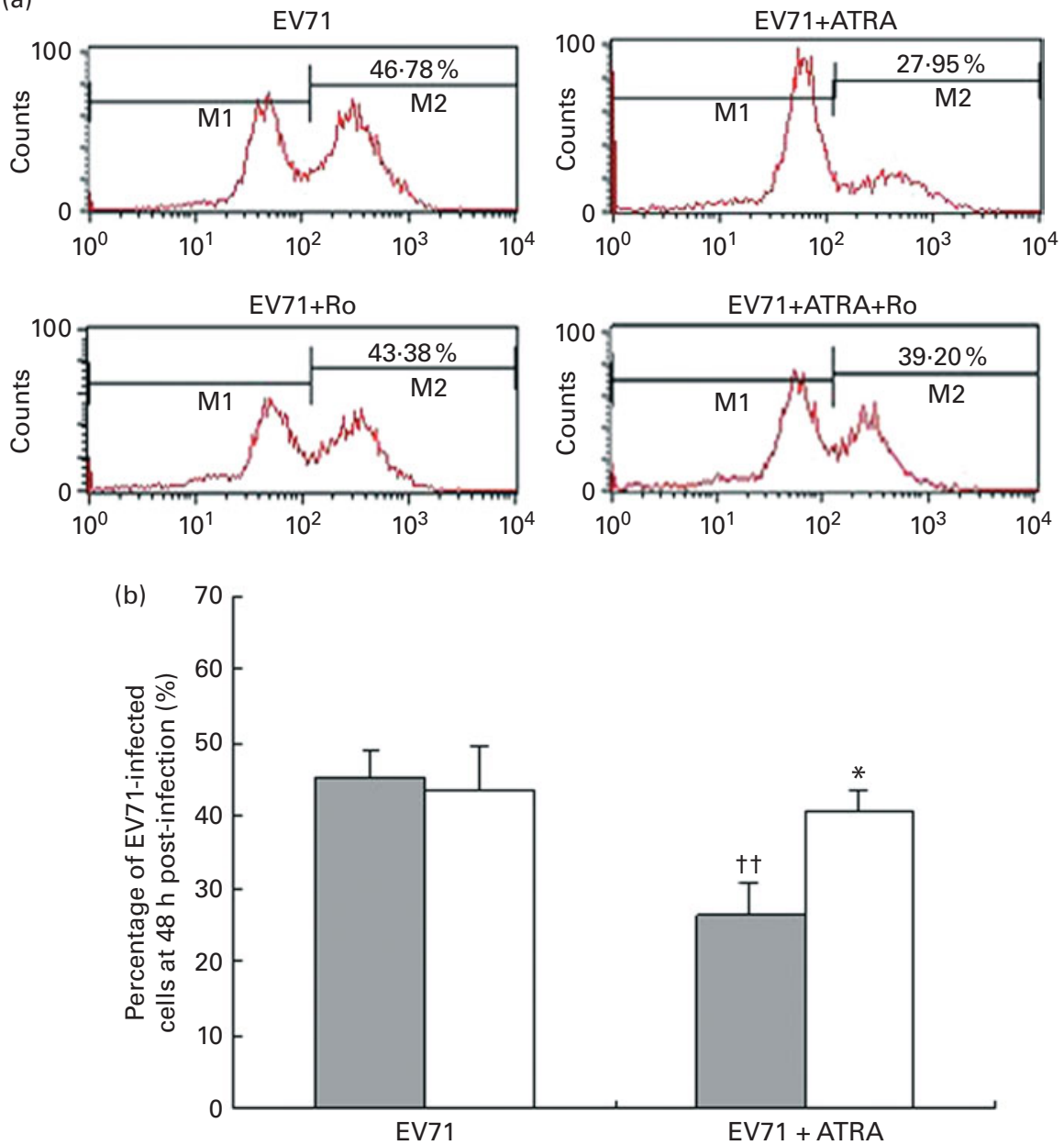

Fig. 2. Enterovirus 71 (EV71)-infected cell count using viral capsid protein 1 (VP1) antibody and flow cytometry. (a) Quantification of EV71-infected cells using flow cytometry. M1 indicates the distribution of fluorescence on cells without EV71 infection and M2 indicates the distribution of fluorescence on EV71-infected cells. (b) Percentage of EV71-infected U937 cells treated with all-trans-retinoic acid (ATRA) 48h after infection. $\square$, without Ro; $\square$, with Ro. Values are means, with standard deviations represented by vertical bars. ${ }^{*}$ Mean value was significantly different from that of the EV71+ATRA group incubated in the absence of Ro $(P<0.05)$. †† Mean value was significantly different from that of the EV71 group incubated in the absence of Ro $(P<0.01)$.

All-trans-retinoic acid reduced the cell apoptosis induced by enterovirus 71 infection

Cell apoptosis induced by EV71 infection was examined by Annexin V/propidium iodide staining $24 \mathrm{~h}$ after infection. The results of three independent experiments are shown in Fig. 3. A very low percentage of apoptotic cells were detected by flow cytometry in the control group, and we did not find any changes in cell apoptosis when U937 cells were treated with ATRA alone. Notably, EV71 infection induced a significantly increased percentage of apoptotic cells than of the control cells $(P<0.001)$. More importantly, when ATRA was present in the culture, the percentage of apoptotic cells induced by EV71 declined. However, Ro 41-5253 partly counteracted the action of ATRA. Taken together, these observations suggest that ATRA may protect cells against EV71-induced apoptosis and that RAR- $\alpha$ is involved in this action.

\section{All-trans-retinoic acid increased the production of interferon- $\alpha$ induced by enterovirus 71 infection}

Type I IFN are potent antiviral cytokines that can be regulated by retinoids in vitro in some cell models ${ }^{(13,21)}$. To determine the effect of ATRA on the production of type I IFN induced by EV71, we measured the production of IFN- $\alpha$ at both mRNA and protein levels in our cell model. As shown in Fig. 4(a), ATRA treatment alone resulted in a marked upregulation of $I F N-\alpha 1$ mRNA expression at $24 \mathrm{~h}$. By contrast, EV71 infection alone decreased the expression of $I F N-\alpha 1$ mRNA. Moreover, when ATRA was added to the EV71-infected cell culture, the expression of $I F N-\alpha 1$ mRNA was significantly increased compared with that in cells treated with ATRA treatment $(P<0 \cdot 05)$. A similar pattern of IFNAR1 (a component of type I IFN receptor) mRNA expression was also observed (Fig. 4(b)). These observations were further confirmed by ELISA. After treatment of EV71-infected cells with ATRA 

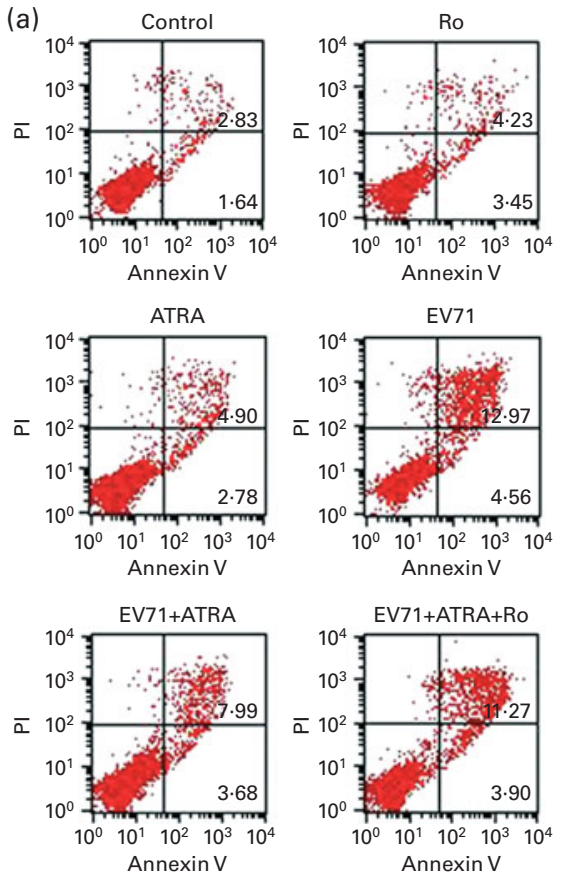

(b)

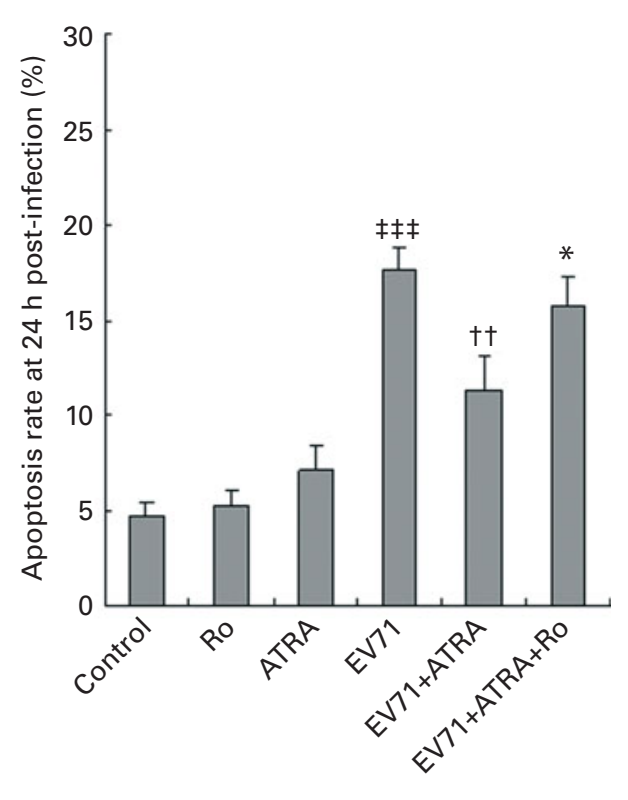

Fig. 3. Effect of all-trans-retinoic acid (ATRA) on apoptosis induced by enterovirus 71 (EV71) infection. Cells were infected with EV71 at a multiplicity of infection of 10. (a) Apoptosis ratio detected by flow cytometry in U937 cells $24 \mathrm{~h}$ after infection. Viable cells were Annexin $\mathrm{V}$ negative and propidium iodide (PI) negative (lower left quadrant) and necrotic cells were Annexin $V$ negative and PI positive (upper left quadrant). Apoptotic cells were differentiated as those in early apoptosis (Annexin $\mathrm{V}+/ \mathrm{PI}-$, lower right quadrant) and late apoptosis (Annexin $\mathrm{V}+/ \mathrm{PI}+$, upper right quadrant). (b) Apoptosis rate $24 \mathrm{~h}$ after infection. Total cell apoptosis was calculated by including cells that were in stages of early and late apoptosis. ATRA treatment significantly decreased the percentage of apoptotic cells induced by EV71. Ro, retinoic acid receptor- $\alpha$ antagonist. Values are means, with standard deviations represented by vertical bars. ${ }^{\star}$ Mean value was significantly different from that of the EV71+ATRA group $(P<0.05)$. †† Mean value was significantly different from that of the EV71 group $(P<0.01)$. $\ddagger \ddagger \ddagger$ Mean value was significantly different from that of the control group $(P<0.001)$.

for $48 \mathrm{~h}$, the production of IFN- $\alpha$ at the protein level was significantly higher than that in the control cells $(P<0.001$; Fig. 4(c)). However, Ro 41-5253 treatment successfully blocked the induction of IFN- $\alpha$ mRNA and IFN- $\alpha$ protein expression by ATRA and EV71 infection. These results indicated that ATRA could induce the production of IFN- $\alpha$ in EV71-infected cells, which may contribute to the antiviral effect of ATRA, and that this effect is likely to be mediated by RAR- $\alpha$ activity.

\section{All-trans-retinoic acid regulated the expression of retinoic acid receptor- $\alpha$ and retinoid-induced gene I signalling genes in enterovirus 71-infected cells}

Because the present results indicated that RAR- $\alpha$ was crucial for the antiviral effect of ATRA in vitro, we sought to investigate the modulation of $R A R-\alpha$ mRNA expression by ATRA in EV71-infected cells. The results of fluorescence quantitative PCR revealed that ATRA induced a significant up-regulation of $R A R-\alpha$ mRNA expression in both EV71-infected and noninfected U937 cells. However, when Ro 41-5253 was added to the cell culture, ATRA was unable to increase the expression of $R A R-\alpha$ mRNA. Furthermore, EV71 infection itself had no visible effect on the expression of $R A R-\alpha$ mRNA (Fig. 5(a)).

$R I G-I$ is a classical IFN-stimulated gene involved in the induction of IFN- $\alpha$ production in response to $\mathrm{EV} 71^{(22,23)}$. To further investigate the antiviral effect of ATRA on IFN- $\alpha$ signalling, we measured the expression of RIG-I mRNA and genes involved in the downstream signalling pathway. In untreated U937 cells, RIG-I mRNA was expressed at low levels. ATRA treatment alone resulted in a moderate increase in the expression of RIG-I mRNA, while EV71 infection alone had no discernible effect on the expression of $R I G-I$ mRNA in this cell line. Importantly, the U937 cells infected with EV71 and treated with ATRA exhibited higher levels of RIG-I mRNA expression than those treated with ATRA alone $(P<0.05$; Fig. 5(b)). Additionally, in the ATRA-treated and EV71-infected U937 cell model, we also observed the up-regulation of the expression of several downstream genes (IFN promoter-stimulating factor 1 (IPS-1), TNF receptor-associated factor 3 (TRAF3), TRAF family member-associated NF-кB activator-binding kinase 1 (TBK1) and interferon regulatory factor 3 (IRF3)) in the RIG-I signalling pathway (Fig. 5(c)-(f)). These findings demonstrate that ATRA treatment can activate RIG-I signalling in EV71-infected cells. The induction of RIG-I mRNA expression by treatment of EV71-infected cells with ATRA was blocked by exposure to Ro 41-5253, suggesting that the up-regulation of the expression of this gene is also mediated via RAR- $\alpha$.

\section{Discussion}

Since 1988, the $\mathrm{WHO}^{(24)}$ has recommended high-dose VA treatment for acute measles virus infection in many resourcepoor settings. In most of the developing countries, VA is also routinely given as a nutritional supplement to reduce the 

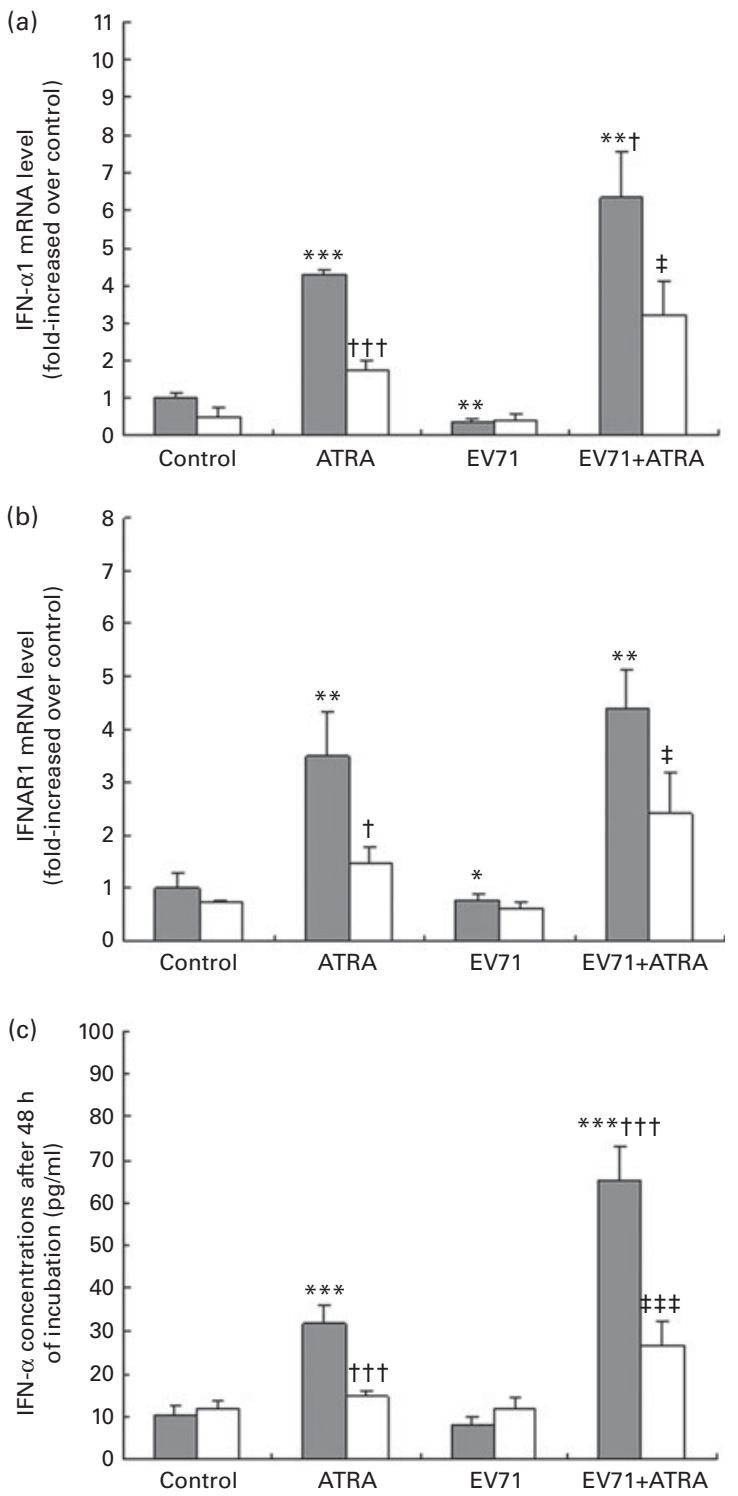

Fig. 4. Induction of interferon (IFN)- $\alpha$ expression. U937 cells were infected with enterovirus 71 (EV71) at a multiplicity of infection of 10 in the presence of $1 \mu \mathrm{M}$-all-trans-retinoic acid (ATRA) with ( $\square$ ) or without ( $\square$ ) $10 \mu \mathrm{M}$-Ro 41-5253 (retinoic acid receptor- $\alpha$ antagonist). (a) IFN- $\alpha 1$ gene expression assessed using quantitative real-time PCR $24 \mathrm{~h}$ after treatment. (b) IFN- $\alpha$ receptor 1 (IFNAR1) gene expression assessed using quantitative real-time PCR $24 \mathrm{~h}$ after treatment. (c) IFN- $\alpha$ levels were detected after $48 \mathrm{~h}$ of incubation using an ELISA. Values are means, with standard deviations represented by vertical bars. Mean value was significantly different from that of the control group incubated in the absence of Ro 41-5253: ${ }^{\star} P<0.05,{ }^{\star *} P<0.01,{ }^{\star \star \star} P<0.001$. Mean value was significantly different from that of the ATRA group incubated in the absence of Ro 41-5253: $† P<0.05$, t†† $P<0.001$. Mean value was significantly different from that of the EV71+ATRA group incubated in the absence of Ro 41-5253: $\ddagger P<0.05$, $\ddagger \ddagger \ddagger P<0.001$.

overall risk of morbidity and mortality ${ }^{(25,26)}$. Although nonspecific effects on immune function and/or epithelial repair have been suggested, the mechanism by which this vitamin acts to prevent infection is not yet fully understood ${ }^{(27)}$.

The potential impact of ATRA on viral replication in vitro has been examined previously by several groups ${ }^{(7,9,28)}$. However, there has been no study to date reporting the antiviral effect of ATRA on EV71 infection. In the present study, we found that ATRA can protect cells from EV71 infection and can partially rescue infected cells from EV71-induced apoptosis. These observations indicate that ATRA has an antiviral action against EV71. However, as only one strain isolated from a patient was used in the present study, as to whether the antiviral effect of ATRA on EV71 is strain specific deserves further study.

In addition, data obtained from the in vitro study also demonstrated that ATRA could significantly increase the production of IFN- $\alpha$ in EV71-infected cells. IFN- $\alpha$ is one of the most important antiviral cytokines in innate immune response. The early induction and action of type I IFN result in cellular resistance to viral infection and inhibition of viral replication and dissemination ${ }^{(29)}$. A previous study has shown that IFN has a protective role in EV71 infection. In a mouse model of EV71 infection, the induction of type I IFN by polyinosinicpolycytidylic acid (poly(I:C)) was found to reduce viral loads. Conversely, the administration of IFN-neutralising antibody facilitated EV71 infection and exacerbated disease severity ${ }^{(30)}$ In our cell model, EV71 failed to induce the production of IFN- $\alpha$, which may contribute to the pathogenesis of EV71 infection. Notably, ATRA exhibits a significant IFN-inducing effect in EV71-infected cells, effectively counteracting EV71's strategy for escaping the innate immune response of the host.

ATRA has been shown to induce certain elements of IFN signalling in some in vitro models, including RIG-I ${ }^{(11,13,21)}$. $R I G-I$ is an important IFN-stimulated gene, and it can induce the expression of IFN via IPS-1. IPS-1 is an adaptor involved in RIG-I-mediated antiviral immune responses ${ }^{(31)}$. The interaction between activated RIG-I and IPS-1 induces the recruitment of downstream signalling molecules, including TRAF3, followed by the activation of TBK1 and inhibitor of NF- $\mathrm{BB}$ kinase $\varepsilon$. These kinases then activate IRF3, an essential transcription factor regulating the expression of type I IFN during virus infection; finally, these molecules induce the production of type I IFN ${ }^{(32)}$. In the present study, we found that the expression of $R I G-I$ mRNA and its downstream signalling genes was up-regulated in EV71-infected cells treated with ATRA. Therefore, it can be suggested that the effect of ATRA on the anti-EV71 immune response is modulated by the RIG-I signalling pathway.

Retinoid is known to exert most of its biological effects via nuclear retinoid receptor signalling ${ }^{(33)}$. There are two subfamilies of retinoid receptors, the RAR and the retinoid X receptors, and each has three members: $\alpha ; \beta ; \gamma^{(34)}$. These receptors can form heterodimers with retinoid $\mathrm{X}$ receptor or homodimers with themselves and then bind to retinoic acid-responsive elements or retinoid $\mathrm{X}$ receptor elements to control the expression of retinoic acid-responsive genes in the presence of retinoids ${ }^{(35)}$. The results of our preliminary experiment indicated that RAR- $\alpha$ was a main receptor for ATRA in U937 cells; thus, a selective RAR- $\alpha$ antagonist, Ro 41-5253, was used to investigate the pathway of ATRA action in EV71-infected cells. The results of the in vitro study indicated that all the effects of ATRA on EV71-infected cells (including the reduction of the percentage of infected and apoptotic cells, induction of the production of IFN- $\alpha$ and up-regulation of the expression of RIG-I and its downstream signalling 

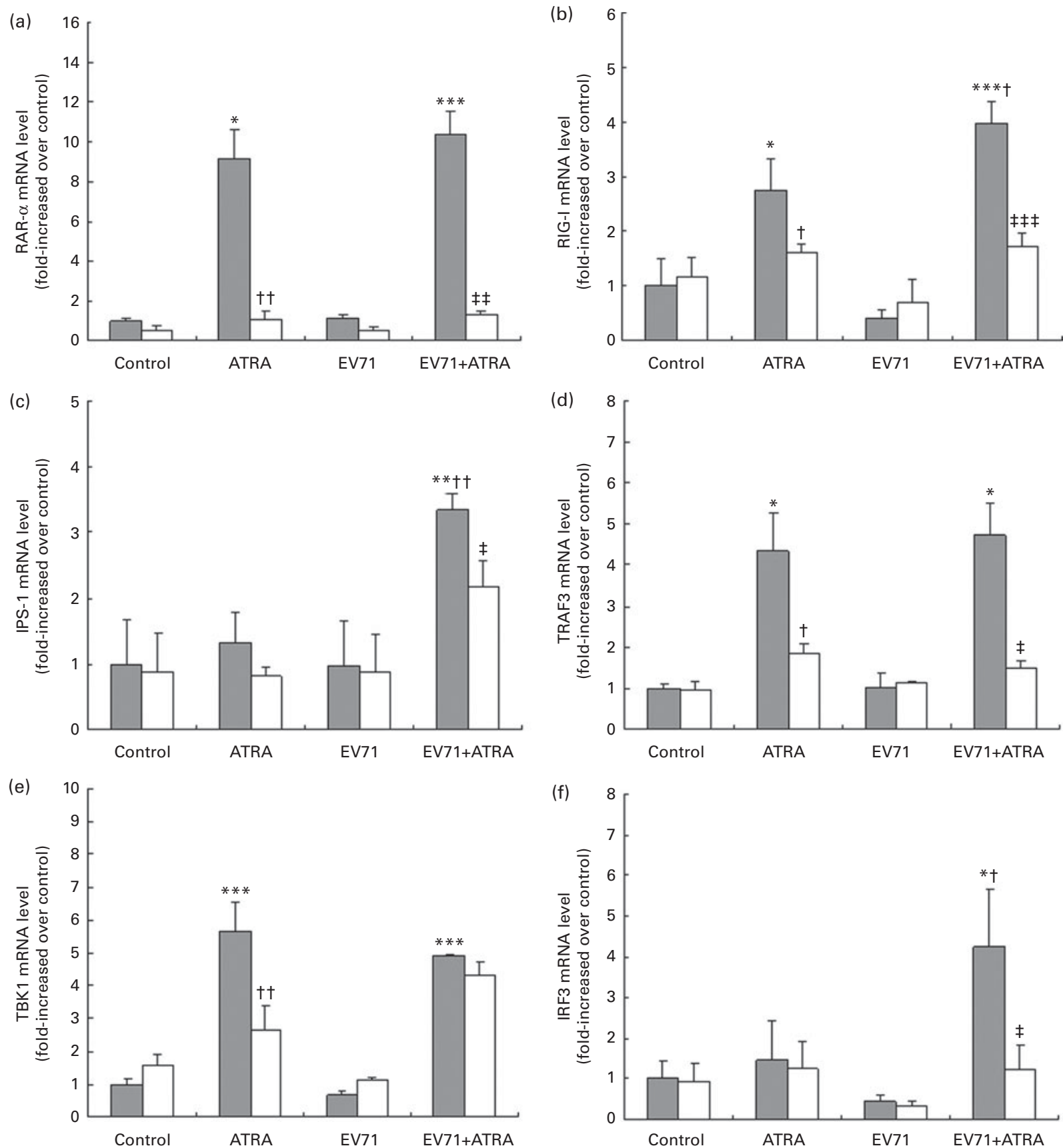

Fig. 5. Gene expression regulated by all-trans-retinoic acid (ATRA) in enterovirus 71 (EV71)-infected cells. U937 cells were infected with EV71 at a multiplicity of

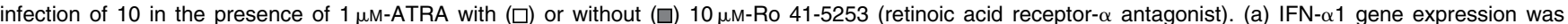
assessed using quantitative real-time PCR $24 \mathrm{~h}$ after treatment. (b) IFN- $\alpha$ receptor 1 (IFNAR1) gene expression was assessed using quantitative real-time PCR $24 \mathrm{~h}$ after treatment. (c) IFN- $\alpha$ levels were detected after $48 \mathrm{~h}$ of incubation using an ELISA. Values are means, with standard deviations represented by vertical bars. Mean value was significantly different from that of the control group incubated in the absence of Ro 41-5253: ${ }^{*} P<0.05,{ }^{* \star} P<0.01,{ }^{* \star \star} P<0.001$. Mean value was significantly different from that of the ATRA group incubated in the absence of Ro 41-5253: $\uparrow P<0.05, \dagger \dagger P<0.01$. Mean value was significantly different from that of the EV71+ATRA group incubated in the absence of Ro 41-5253: $\ddagger P<0.05, \ddagger \ddagger P<0.01$, $\ddagger \ddagger \nexists P<0.001$.

genes) were inhibited at different levels by Ro 41-5253. These findings indicate that the effects of ATRA on EV71 infection were likely to be mediated via the RAR- $\alpha$ pathway. However, Ro 41-5253 did not completely reverse all the effects of ATRA, which raises the possibility that other retinoid receptors may also contribute modestly to the antiviral effect. Further studies are needed to elucidate these findings.
In conclusion, we have demonstrated that ATRA is a potent IFN inducer that effectively inhibits EV71 and significantly regulates the RIG-I signalling pathway in the human monocytic cell line. The antiviral effect of ATRA appears to occur through a RAR- $\alpha$ pathway. The present results raise the possibility that ATRA may directly contribute to anti-EV71 infection by reinforcing innate immunity. 


\section{Acknowledgements}

The present study was supported by a grant from the Ming Dao project of Fudan University (MDJH2012020).

The authors' contributions are as follows: Y. Y. and W. W. were responsible for study conception and design; S. C., L. S. and J. X. conducted the research; S. C. analysed the data and wrote the manuscript; Y. Y. and W. W. revised the manuscript; W. W. had primary responsibility for the final content; all the authors read and approved the final manuscript.

None of the authors has any financial and personal relationships with other people or organisations or any other conflicts of interest that could inappropriately influence the present study.

\section{References}

1. Yang F, Ren L, Xiong Z, et al. (2009) Enterovirus 71 outbreak in the People's Republic of China in 2008. J Clin Microbiol 47, 2351-2352.

2. Ni H, Yi B, Yin J, et al. (2012) Epidemiological and etiological characteristics of hand, foot, and mouth disease in Ningbo, China, 2008-2011. J Clin Virol 54, 342-348.

3. Zeng M, El Khatib NF, Tu S, et al. (2012) Seroepidemiology of enterovirus 71 infection prior to the 2011 season in children in Shanghai. J Clin Virol 53, 285-289.

4. Wang Y, Feng Z, Yang Y, et al. (2011) Hand, foot, and mouth disease in China: patterns of spread and transmissibility. Epidemiology 22, 781-792.

5. Mullin GE (2011) Vitamin A and immunity. Nutr Clin Pract 26, 495-496.

6. Chen S, Yang Y, Yan X, et al. (2012) Influence of vitamin A status on the antiviral immunity of children with hand, foot and mouth disease. Clin Nutr 31, 543-548.

7. Trottier C, Chabot S, Mann KK, et al. (2008) Retinoids inhibit measles virus in vitro via nuclear retinoid receptor signaling pathways. Antiviral Res 80, 45-53.

8. Hanley TM, Kiefer HL, Schnitzler AC, et al. (2004) Retinoiddependent restriction of human immunodeficiency virus type 1 replication in monocytes/macrophages. $J$ Virol $\mathbf{7 8 ,}$ $2819-2830$.

9. Isaacs CE, Kascsak R, Pullarkat RK, et al. (1997) Inhibition of herpes simplex virus replication by retinoic acid. Antiviral Res 33, 117-127.

10. Pomponi F, Cariati R, Zancai P, et al. (1996) Retinoids irreversibly inhibit in vitro growth of Epstein-Barr virusimmortalized B lymphocytes. Blood 88, 3147-3159.

11. Soye KJ, Trottier C, Richardson CD, et al. (2011) RIG-I is required for the inhibition of measles virus by retinoids. PLoS One 6, e22323.

12. Luo XM \& Ross AC (2005) Physiological and receptorselective retinoids modulate interferon gamma signaling by increasing the expression, nuclear localization, and functional activity of interferon regulatory factor-1. $J$ Biol Chem 280, 36228-36236.

13. Dimberg A, Nilsson K \& Oberg F (2000) Phosphorylationdeficient Stat1 inhibits retinoic acid-induced differentiation and cell cycle arrest in U-937 monoblasts. Blood 96, $2870-2878$.

14. Lei X, Liu X, Ma Y, et al. (2010) The 3C protein of enterovirus 71 inhibits retinoid acid-inducible gene I-mediated interferon regulatory factor 3 activation and type I interferon responses. J Virol 84, 8051-8061.
15. Wang SM, Chen IC, Su LY, et al. (2010) Enterovirus 71 infection of monocytes with antibody-dependent enhancement. Clin Vaccine Immunol 17, 1517-1523.

16. Gianni M, Terao M, Fortino I, et al. (1997) Stat1 is induced and activated by all-trans retinoic acid in acute promyelocytic leukemia cells. Blood 89, 1001-1012.

17. Yang ZH, Zhu QR, Li XZ, et al. (2005) Detection of enterovirus 71 and coxsackievirus A16 from children with hand, foot and mouth disease in Shanghai, 2002. Zhonghua Er Ke Za Zbi 43, 648-652.

18. Wei D, Yang Y \& Wang W (2007) The expression of retinoic acid receptors in lymph nodes of young children and the effect of all-trans-retinoic acid on the B cells from lymph nodes. J Clin Immunol 27, 88-94.

19. Dong P, Tao Y, Yang Y, et al. (2010) Expression of retinoic acid receptors in intestinal mucosa and the effect of vitamin A on mucosal immunity. Nutrition 26, 740-745.

20. Zhou X, Wang W \& Yang Y (2008) The expression of retinoic acid receptors in thymus of young children and the effect of all-trans-retinoic acid on the development of $\mathrm{T}$ cells in thymus. J Clin Immunol 28, 85-91.

21. Luo XM \& Ross AC (2006) Retinoic acid exerts dual regulatory actions on the expression and nuclear localization of interferon regulatory factor-1. Exp Biol Med (Maywood) 231, 619-631.

22. Lu J, Yi L, Zhao J, et al. (2012) Enterovirus 71 disrupts interferon signaling by reducing the level of interferon receptor 1 . J Virol 86, 3767-3776.

23. Huang HI, Weng KF \& Shih SR (2012) Viral and host factors that contribute to pathogenicity of enterovirus 71 . Future Microbiol 7, 467-479.

24. World Health Organization (1987) Joint WHO/UNICEF statement on vitamin A for measles. Wkly Epidemiol Rec 62 , $133-134$.

25. Imdad A, Herzer K, Mayo-Wilson E, et al. (2010) Vitamin A supplementation for preventing morbidity and mortality in children from 6 months to 5 years of age. The Cochrane Database of Systematic Reviews, issue D8524.

26. Benn CS, Martins C, Rodrigues A, et al. (2005) Randomised study of effect of different doses of vitamin A on childhood morbidity and mortality. BMJ 331, 1428-3142.

27. Mora JR, Iwata M \& von Andrian UH (2008) Vitamin effects on the immune system: vitamins $\mathrm{A}$ and $\mathrm{D}$ take centre stage. Nat Rev Immunol 8, 685-698.

28. Ghazal P, DeMattei C, Giulietti E, et al. (1992) Retinoic acid receptors initiate induction of the cytomegalovirus enhancer in embryonal cells. Proc Natl Acad Sci U S A 89, 7630-7634.

29. Alsharifi M, Mullbacher A \& Regner M (2008) Interferon type I responses in primary and secondary infections. Immunol Cell Biol 86, 239-245.

30. Liu ML, Lee YP, Wang YF, et al. (2005) Type I interferons protect mice against enterovirus 71 infection. J Gen Virol 86, 3263-3269.

31. Kawai T, Takahashi K, Sato S, et al. (2005) IPS-1, an adaptor triggering RIG-I- and Mda5-mediated type I interferon induction. Nat Immunol 6, 981-988.

32. Takeuchi O \& Akira S (2010) Pattern recognition receptors and inflammation. Cell 140, 805-820.

33. Schug TT, Berry DC, Shaw NS, et al. (2007) Opposing effects of retinoic acid on cell growth result from alternate activation of two different nuclear receptors. Cell 129, 723-733.

34. de Lera AR, Bourguet W, Altucci L, et al. (2007) Design of selective nuclear receptor modulators: RAR and RXR as a case study. Nat Rev Drug Discov 6, 811-820.

35. Pfahl M (1993) Signal transduction by retinoid receptors. Skin Pharmacol 6, Suppl. 1, 8-16. 